\title{
- Antiviral drug discovery and development
}

New drugs have transformed the treatment of viral infections - in particular, HIV — in the past two decades. Our interviewees this month describe the rewards and challenges of their complementary roles in antiviral drug discovery and development.

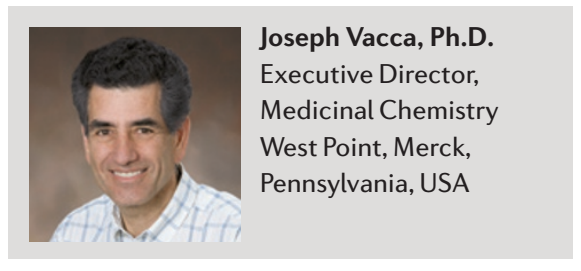

In the mid-1990s, a new class of antiviral drugs known as HIV protease inhibitors revolutionized the treatment of HIV infection. Being involved in this transformation has been the best career experience for Joseph Vacca, Executive Director of the Department of Medicinal Chemistry at Merck, who was the project leader and one of the co-inventors of the firstgeneration HIV protease inhibitor indinavir. "It was thrilling to see how HIV went from being a death sentence to being a chronic, manageable disease for many people," recalls Vacca. "Many better treatments now exist, but I feel that indinavir helped to show people what a potent drug could do, and also taught people how to make better ones."

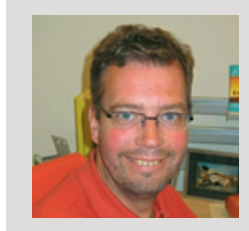

David M. Burger, Pharm.D., Ph.D. Associate Professor, Department of Clinical Pharmacy and Nijmegen University Centre for Infectious diseases (NUCl), Radboud University Nijmegen Medical Centre, The Netherlands

Variation in drug pharmacokinetics can pose a major challenge to the optimal use of drugs for HIV in different patient populations.

“Antiretroviral agents are well known for their susceptibility to drug-drug interactions, a narrow therapeutic range and wide interpatient variability," says David Burger, a clinical pharmacologist at the Nijmegen University Centre for Infectious Diseases in the Netherlands. This variability means that treatment regimes that have been developed largely on the basis of clinical trials in patient populations in Europe and the United States, for example, might not be appropriate for patients in countries in Asia and Africa. Furthermore, many of these countries may be lacking the expertise in clinical pharmacology to investigate and implement adjustments that are needed in treatment regimes.
Vacca's scientific career began with Peter Lansbury Sr at The State University of New York at Buffalo, USA. "I chose organic chemistry because I enjoyed the challenge of lab work and the gratification of planning a synthetic sequence that resulted in a product," he says. When he was searching for a position during the last year of his graduate studies, he sent his résumé to an old lab colleague who was working at Merck, and was offered a position at the West Point site. "Medicinal chemistry appealed to me because of my interest in biology and the chance of being able to discover a drug. At Merck I could continue to do high level synthetic chemistry and was encouraged to publish in the scientific literature," he explains.

For 12 years, he worked in the laboratory as a synthetic chemist on projects ranging from $\alpha_{2}$ adrenoceptor antagonists for depression, the synthesis of myo-inositol polyphosphates to study cell signalling pathways and finally to HIV protease inhibitors before moving to a managerial role. "My attraction to managing a group was to be involved with several drug discovery projects

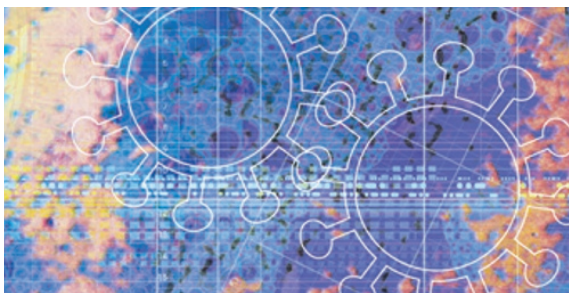

and to be able to interact with many high level scientists within our department and across the organization," says Vacca. Now, he oversees many of the Department's drug discovery projects and acts as mentor and consultant to the chemistry heads who report to him. It is this interaction with talented colleagues that he most enjoys. "It is a pleasure to come in every day and see what kinds of new discoveries have been made," Vacca says.

Of course, given the high attrition rates in drug discovery and the 26 years he has been at Merck, Vacca is familiar with the ongoing challenges in the field. "I have personally failed at every point in the path to a drug and it is usually from something unexpected." Still, successfully tackling these challenges is very rewarding. "I am excited to see clinical data from our compounds and to see what effect they have on the lives of patients," says Vacca. "I was lucky enough to be the chemistry director when we started our recent integrase project [which has now resulted in the approval of the first HIV integrase inhibitor, raltegravir] and can see once again how a compound with a new mechanism of action affects the lives of people."
To help address this problem, a pharmacokinetic unit was created in 2002 at the Thai Red Cross AIDS Research Centre through the HIV Netherlands Australia Thailand research collaboration (HIV-NAT, http://www.hivnat.org) founded in 1996. "Here, we've built the infrastructure for conducting clinical pharmacology research on antiretroviral therapy in HIV-negative and HIV-positive Thai subjects," says Burger, who remains involved in pharmacokinetic studies that are performed at HIV-NAT. The unit now boasts both a clinical research section and a well-equipped pharmacology laboratory to enable research such as determining appropriate dosing of antiretrovirals in all populations, including paediatrics, evaluating drug-drug interactions, establishing bioequivalence of generic compounds and pharmacogenetics.

Burger's interest in the clinical pharmacology of antiviral and antimicrobial agents began after completing his Ph.D. on the clinical pharmacokinetics of antiretroviral agents. While working as a hospital pharmacist resident, one of his former supervisors contacted him about a position at the Nijmegen Medical Centre, and the opportunity to work both nationally and internationally on a subject in which clinical pharmacology can contribute to optimized treatment of patients was what attracted him to work there. "The Department of Clinical Pharmacy had a stimulating and motivating vision on the importance of clinical pharmacology research in infectious diseases," says Burger. He now directs a research group working on the clinical pharmacology of antimicrobial agents, with a focus on HIV, tuberculosis and fungal infections.

In addition to their research, his group is also involved in in-patient and out-patient clinics, as well as teaching (bio)medical and pharmacy students. "With increased access to antiretroviral agents for patients in developing countries, our clinical pharmacological expertise can be used for training and operational research in areas where it is most needed," says Burger. "Most rewarding to me is the training of young researchers and sharing our knowledge and expertise in parts of the world where clinical pharmacologists do not exist. Working in study teams together with medical doctors, pharmacists and nurses clearly improves the scientific outcome of our work."

WEB SITE

For more Career snapshots, please see:

Career snapshots: http://www.nature.com/naturejobs/ $\underline{\text { magazine/career_snaps.html }}$ 